\title{
Effect of Recycled Waste Brick Fine Aggregate on Compressive Strength and Flexural Strength of Mortar
}

\author{
Xian LI, Fujin WANG, Fei LI \\ Beijing Collaborative Innovation Center for Energy Conservation and Emissions Reduction \\ Technology, Beijing University of Civil Engineering and Architecture, Beijing 100044, China
}

Keywords: recycled brick aggregate; compressive and flexural strength; microstructure;

Abstract: Recycled waste brick (RWB) aggregate has obvious different properties from recycled concrete (RC) aggregate and is hardly accepted because of its lower strength and higher water absorption. The effect of recycled waste brick fine aggregate on compressive strength and flexural strength of mortar was studied. Meanwhile the influence of recycled waste brick fine aggregate content and water to cement ratio (w/c) was considered in the experiment. Optical microscope with a large depth-of-field was used to observe the microstructure of hardened mortar made with manufactured sand and recycled fine aggregate. The results show that the compressive strength and flexural strength of mortar decreased with the increase of recycled fine aggregate content in various w/c. The recycled aggregate has inner-curing effect, which can benefit the hydration of cement as well as the interfacial transition zone between the hydration product and aggregate.

\section{Introduction}

With the acceleration of the urbanization in China, the huge amount of construction and demolished waste seriously threatens the environmental safety. Researchers have paid attention to the recycling use of recycled concrete for a long time. As one of the main type of the demolished waste, waste brick can also be broken into coarse and fine aggregate. However, Recycled waste brick (RWB) aggregate has obvious different properties from recycled concrete (RC) aggre gate and is hardly accepted because of its low strength and high water absorption.

A few researches on RWB aggregate mainly concentrated in the influence of the waste brick aggregate on the performance of concrete ${ }^{[1-4]}$. The influence is analyzed on the mechanical properties and the microstructure of recycled concrete ${ }^{[5]}$. The main factors affecting the tensile strength and the resistance to salt freeze are obtained. In this paper, the effects of different amount of RWB fine aggregate and different w/c on the compressive strength and flexural strength of mortar are studied.

\section{Raw materials and experiment design}

Raw materials

P-O 42.5 cement from JinYu Group and polycarboxylate superplasticizer (SP) were used. Fine aggregate includes three types, ISO standard sand(SS), manufactured sand(MS) and recycled waste brick sand (RS), shown in Figure 1 to Figure 3. The packing density of MS and RS is $1596 \mathrm{~kg} / \mathrm{m}^{3}$ and $1220 \mathrm{~kg} / \mathrm{m}^{3}$ respectively. And the water demand ratio of RS is 1.44 according to Chinese Standard GB/T 25176-2010. 


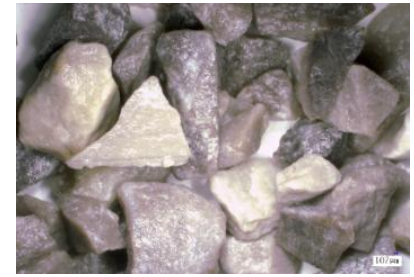

Fig. 1. Manufactured sand (MS)

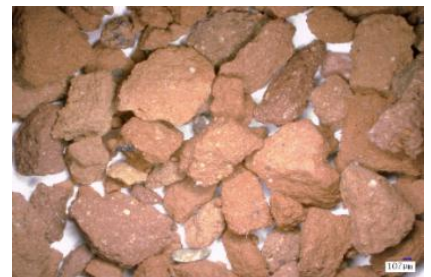

Fig. 2. Recycled sand (RS)

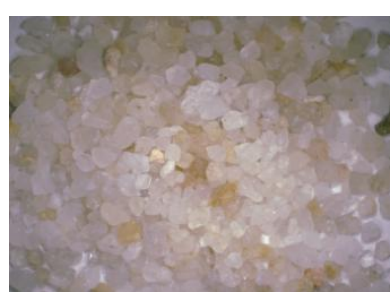

Fig. 3. ISO Standard sand (SS)

Mix proportion design

The influence of w/c and fine aggregate type has been designed in the experiment. The amount of cement, fine aggregate and superplasticizer content are fixed in each w/c mixture. The proportion is shown in Table 1, where 0.4ISO means w/c is 0.4 , the fine aggregate use SS and 0.4RX means $\mathrm{w} / \mathrm{c}$ is $0.4, \mathrm{x} \% \mathrm{MS}$ is substitute by RS.

Table 1. Proportions of the mortar $(\mathrm{g})$

\begin{tabular}{cccccc}
\hline NO. & water & cement & SS & MS & RS \\
\hline 0.4ISO & 180 & 450 & 1350 & 0 & 0 \\
0.4R0 & 180 & 450 & 0 & 1350 & 0 \\
0.4R50 & 180 & 450 & 0 & 675 & 675 \\
0.4R100 & 180 & 450 & 0 & 0 & 1350 \\
0.5ISO & 225 & 450 & 1350 & 0 & 0 \\
0.5R0 & 225 & 450 & 0 & 1350 & 0 \\
0.5R50 & 225 & 450 & 0 & 675 & 675 \\
0.5R100 & 225 & 450 & 0 & 0 & 1350 \\
0.6ISO & 270 & 450 & 1350 & 0 & 0 \\
0.6R0 & 270 & 450 & 0 & 1350 & 0 \\
0.6R50 & 270 & 450 & 0 & 675 & 675 \\
0.6R100 & 270 & 450 & 0 & 0 & 1350 \\
\hline
\end{tabular}

Experimental procedure

Standard curing was used in the experiment, that is, the temperature of $(20+1)^{\circ} \mathrm{C}$ and relative humidity greater than $95 \%$ of the standard curing chamber until testing age.

The compressive strength and flexural strength of mortar specimens were prepared by GB/T 17671-1999, the strength test method (ISO method), including 3d, $7 \mathrm{~d}$ and $28 \mathrm{~d}$. The microstructure of MS, RS and SS was observed by KEYENCE VHX-2000 optical microscope with a large depth-of-field. 


\section{Results and discussion}

The results of compressive and flexural strength are shown in the Table 2.

Table 2. Results of compressive and flexural strength (MPa)

\begin{tabular}{ccccccccc}
\hline \multirow{2}{*}{ NO. } & \multicolumn{2}{c}{ compressive strength } & growth & \multicolumn{3}{c}{ flexural strength } & \multicolumn{2}{c}{ growth } \\
& $3 \mathrm{~d}$ & $7 \mathrm{~d}$ & $28 \mathrm{~d}$ & rate & $3 \mathrm{~d}$ & $7 \mathrm{~d}$ & $28 \mathrm{~d}$ & rate \\
\hline 0.4ISO & 5.90 & 8.98 & 10.99 & $22 \%$ & 31.4 & 43.1 & 67.9 & $58 \%$ \\
0.4R0 & 10.62 & 10.00 & 13.62 & $36 \%$ & 52.6 & 57.5 & 83.9 & $46 \%$ \\
0.4R50 & 9.59 & 8.95 & 12.27 & $37 \%$ & 44.7 & 51.2 & 68.0 & $33 \%$ \\
0.4R100 & 6.19 & 7.18 & 9.97 & $39 \%$ & 25.6 & 35.0 & 47.9 & $37 \%$ \\
0.5ISO & 6.21 & 7.85 & 9.78 & $25 \%$ & 29.1 & 39.7 & 55.3 & $39 \%$ \\
0.5R0 & 8.52 & 9.35 & 11.65 & $25 \%$ & 37.4 & 46.0 & 59.3 & $29 \%$ \\
0.5R50 & 5.35 & 8.10 & 10.34 & $28 \%$ & 21.9 & 38.2 & 55.3 & $45 \%$ \\
0.5R100 & 4.18 & 6.58 & 8.81 & $34 \%$ & 15.8 & 31.1 & 42.7 & $38 \%$ \\
0.6ISO & 5.13 & 6.94 & 8.11 & $17 \%$ & 18.8 & 31.1 & 40.1 & $29 \%$ \\
0.6R0 & 5.53 & 7.83 & 9.29 & $19 \%$ & 22.6 & 33.4 & 39.8 & $19 \%$ \\
0.6R50 & 4.62 & 6.30 & 8.04 & $28 \%$ & 17.0 & 26.8 & 38.2 & $43 \%$ \\
0.6R100 & 3.60 & 5.11 & 6.31 & $23 \%$ & 12.8 & 21.1 & 31.4 & $49 \%$ \\
\hline
\end{tabular}

Note: growth rate means the growth of $28 \mathrm{~d}$ strength refer to $7 \mathrm{~d}$ strength.

\section{Compressive strength}

The experimental data were processed with ISO standard sand group as the baseline, and the other groups were compared with each other. The compressive strength ratio of mortar under different $w / c$, different aggregate types and mixing amount was obtained. The results are as shown in Figure 4, 5 and 6.
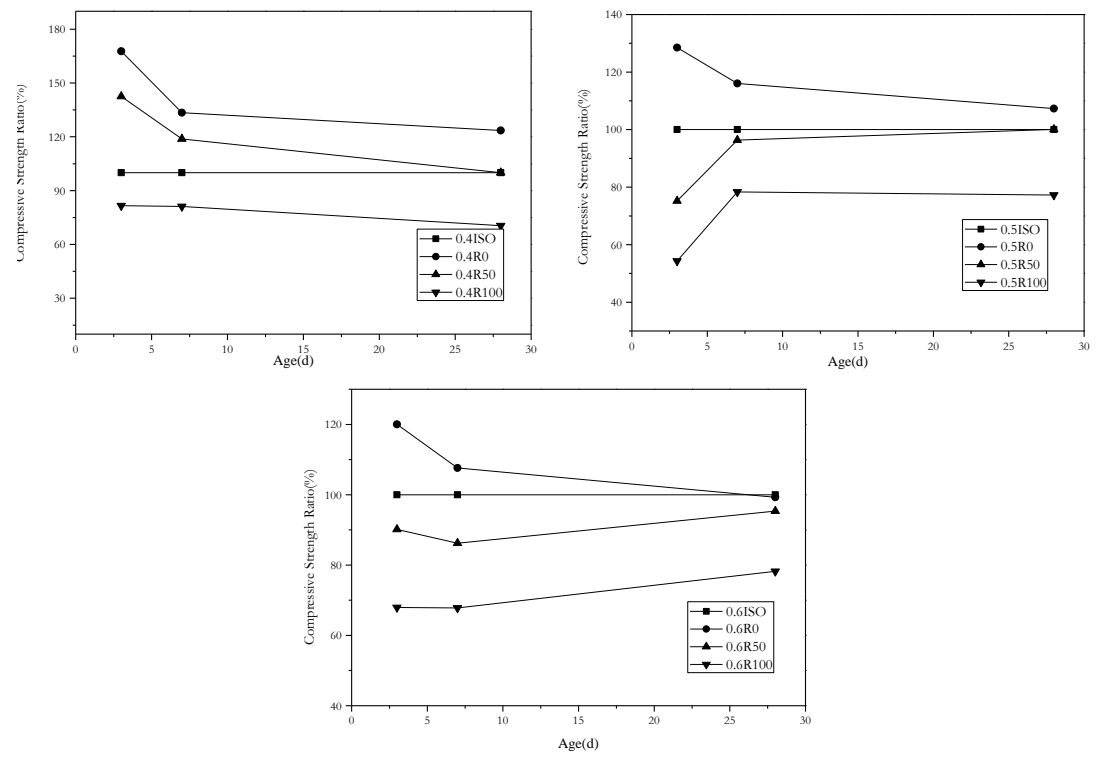

Fig. 4. $w / c=0.4$ mortar

Fig. 5. w/c $=0.5$ mortar

Fig. 6. $w / \mathrm{c}=0.6$ mortar

From Figure 4, 5 and 6, we can see that no matter what the w/c is, the compressive strength of $\mathrm{R} 0$ group is the highest, which is not mixed with RS group, this is due to the high strength of MS, which means the strength of the aggregate plays a decisive role, and then affect the overall strength of mortar. 
When w/c=0.4, the long-term strength of R0, R50, and R100 group growth slowed down, which is showing in figure 4. The early (3d) compressive strength of R0 group was $68 \%$ higher than that of ISO group, and the latter (28d) compressive strength was $24 \%$ higher than that of ISO, R50 group (3d) was improved by $42 \%$.

When $w / c=0.5$ and $w / c=0.6$, similar regulars were found.

Flexural strength

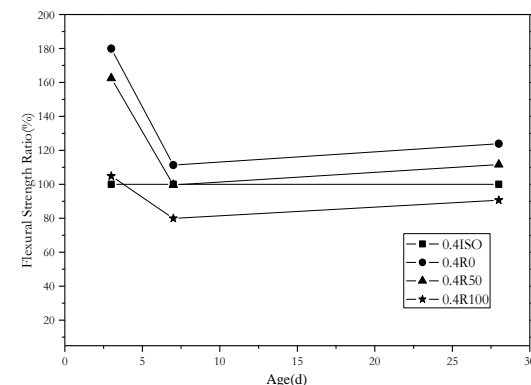

Fig. 7. $w / c=0.4$ mortar

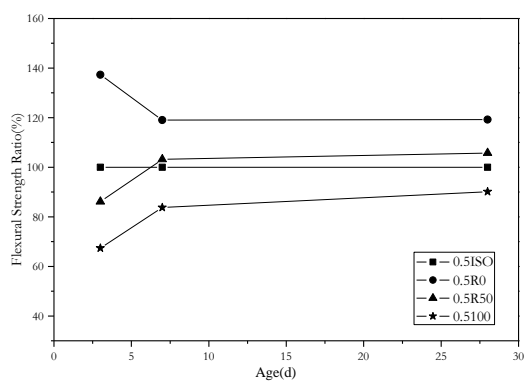

Fig. 8. $w / c=0.5$ mortar

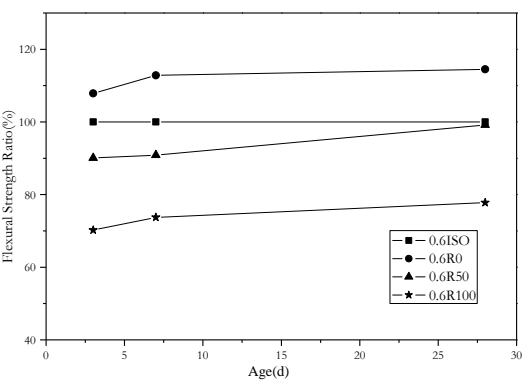

Fig. 9. $w / c=0.6$ mortar

Combined with the figure 7, 8 and 9 , it can be seen that the R0 group is the highest flexural strength, due to the high strength of MS. The later (28d) flexural strength of R50 and R100 group growth have accelerated trend. The rules of flexural strength and compressive strength is very close. When w/c $=0.5$, R50 group 7d, 28d flexural strength were higher than those of SS group, the flexural strength were up $3 \%$ and $6 \%$. And due to the internal curing effect of RS, the later strength has a growth trend.

Micro-morphology

The experimental study found that the cross section of the ordinary mortar is often destroyed at the interface of aggregate and cement paste. The interface microstructure of the aggregate and cement paste is the decisive factors of the strength. The failure of RS mortar are observed both in interface and recycled aggregate because of lower strength of waste brick and its internal damage and micro cracks due to crushing. And large variability of interface causes lower mechanical properties of recycled sand mortar.

The microstructure of MS and RS mortar after 90d curing were observed by optical microscope system, as shown in Figure 10 and 11 respectively. It can be seen that interface between the aggregate and the cement paste is relatively flat in MS mortar than that of RS mortar.

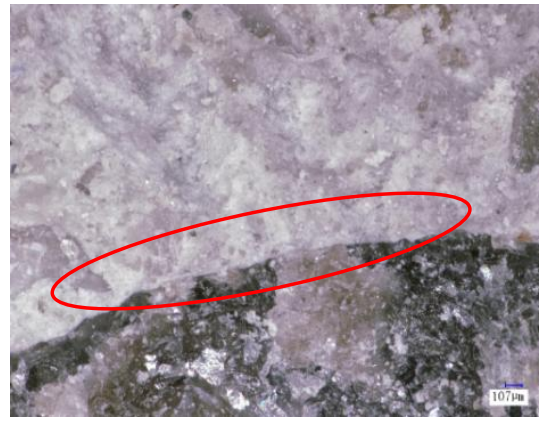

Fig. 10. Manufactured sand mortar solidification

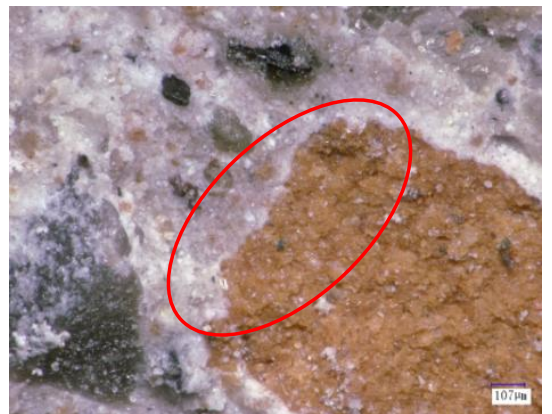

Fig. 11. Recycled sand mortar solidification Due to the loose and porous characteristic of RS, it may adsorb cement slurry at the initial stage of hardening. causing the decrease of w/c near interface. RS gradually release moisture in later stage of the reaction called self-curing, which promote the further hydration reaction of cement and 
enhance the growth of strength in long term. However, the improvement of interface cannot compensate the decrease due to low strength of waste brick RS in this study.

\section{Conclusion}

1) The compressive and flexural strength of mortar specimen decreased with the increase of RS content while w/c ranges from 0.4 to 0.6 . However, the long-term strength growth of RS mortar is more obvious.

2) The inner curing effect of RS promotes the hydration of cement, which makes the interfacial transition zone more dense. The bonding between fine aggregate and hardened paste is stronger, which is beneficial to the further development of strength.

\section{Acknowledgements}

The authors would like to acknowledge the financial support of Beijing Natural Science Foundation (project number: 8144043) and National Natural Science Foundation of China (project number: 51408622).

\section{References}

[1] Q in Liu. Recycled brick coarse aggregate concrete mix proportion and physical performance of [D]. Zhengzhou University, 2010. (In Chinese)

[2] Qi Liang, Tingjian Huang, Juan Zhao, Jinling Ye, Jianzhi Teng. Concrete, 2009, 03:44-46. (In Chinese)

[3] Shuozhi Wang, Hai Li Cheng, Kun Zhou, Dawei Sun, Lei Wang, Guozhi Wei. Renewable resources and circular economy, 2014, 11:28-31. (In Chinese)

[4] Zhao Lan, Shuang Li, Lixin Liu, Tong Hao. New building materials, 2012, 06:30-32+44. (In Chinese)

[5] Fei Li, Xiaoqi Zhang, Huibiao Dong, Jingyun Shen. Concrete, 2015, 02:56-58+62. (In Chinese) 